\title{
Human dermal reticular fibroblasts at confluence display a signature micro pattern in vitro*
}

\author{
Denis E. Solomon \\ 9B Somerville Road, Wigan, Lancs., WN1 2RX, England; denissolomon@yahoo.com
}

Received 16 August 2013; revised 16 September 2013; accepted 1 October 2013

Copyright (C) 2013 Denis E. Solomon. This is an open access article distributed under the Creative Commons Attribution License, which permits unrestricted use, distribution, and reproduction in any medium, provided the original work is properly cited.

\begin{abstract}
This paper sets out to demonstrate that scraping of the flat dorsal surface of human dermis with a scalpel blade and cell plating without centrifugation can lead to the recognition and identification of the individual packing micro pattern of dermal reticular fibroblasts at confluence. The characteristic alignment of papillary and reticular fibroblasts at right angles to each other led to the positive identification of reticular fibroblasts. A non-enzymatic means of sub-culturing (passaging), which yields fully functional, healthy cells with normal, phenotypic morphology is also described. Implications for published subcutaneous wound healing studies are discussed as well as the confluent reticular fibroblast configuration, interpreted as an anatomic site identity code, which may be the address of a specific fibroblast gene pattern expression.
\end{abstract}

Keywords: Papillary and Reticular Fibroblasts; Cell Culture of Human Dermal Fibroblasts; Dermal Filler; Dermal Reticular Fibroblast Micro Pattern; Wound Healing; Anatomic Site Identity Code

\section{INTRODUCTION}

Tissue culture techniques have been traditionally used for garnering yields of dermal fibroblasts. Routine methodology employs enzymatic digestion to isolate cells followed by centrifugation to obtain a cell pellet which is re-suspended before cell plating and incubated to obtain a primary cell culture. Additional enzymatic digestions are used for passaging (sub-culturing) as a means of expanding fibroblast cell lines. Classic cell culture using skin tissue explants does not involve enzyme use but

"Competing interest: The cell culture technique used and described by the author herein is subject to UK (2010) and USA (2011) patent applications. offers no insight into in situ cellular architecture, just the morphology of the cell spread in a loose, unguided semicircular array in vitro. Within the context of this paper, all previously published experimental studies done with human dermal fibroblasts using cultured cell lines or cloned cell lines will be discarded.

The focus on dermal fibroblasts has heightened in the last thirty years with the onset of cultured skin substitutes and in wound healing studies for tissue repair and regeneration. However, the majority of past publications offer little in terms of the delineation between papillary and reticular fibroblasts and even authors of review papers have used the term "fibroblast" in a generic sense [1] as did authors of mathematical modelling during dermal wound healing [2], even though there had been earlier published reports of fibroblast heterogeneity and population subsets $[3,4]$. None of the above has offered any insight into possible micro patterns which may be resident in dermal tissue or the means to regenerate them.

Researchers have not realised that cell culture techniques can be used to gain an insight into in situ cellular architecture [5] from initial primary cell isolates. Human dermal fibroblasts have been isolated by various enzymatic digestions [6,7] of minced dermis (followed by centrifugation, re-suspension of the cell pellet, then plating of cells) or by classic tissue explant experiments [8] the latter authors seeking to distinguish between the two types of dermal fibroblasts.

No author in the published literature has sought to question the time it takes from the acquisition of skin tissue segments to the confluence of the first primary fibroblast isolate. Enzymatic digestion of the epidermis takes hours to be followed by the setting up of tissue explant experiments. More time is lost awaiting the spread of cells from the (now) dermal tissue. Some researchers have used a second enzyme digestion to isolate fibroblasts in an attempt to speed up the process obtaining a mixed yield of papillary and reticular fibroblasts [9]. 
Figure 6 in Schönherr et al. (1993) [10] shows the morphological differences between papillary and reticular dermal fibroblasts from adult skin. However, these fibroblasts were donated (no stated isolation protocol), cultured with $10 \%$ foetal calf serum and used between $\mathrm{P}_{9}$ $\mathrm{P}_{17}$; hence the morphology of the pictured fibroblasts may be questionable. Commercial sources vary in their dermal fibroblast cell culture techniques as do manufacturers of cultured skin substitutes. The use of autologous fibroblasts as a dermal filler is now a viable clinical proposition [11]. It is of paramount importance that those fibroblasts are harvested with a safe, standard, assured cell culture technique (see later comment under Discussion).

\section{MATERIALS AND METHODS}

Cell culture materials including dishes and flasks were obtained as previously described [12]. A Nikon phase contrast microscope was used throughout these studies.

\subsection{Cell Culture Technique}

Fresh skin tissue obtained from the operating theatre was trimmed of subcutaneous fat and prepared for Dispase digestion within an hour of receipt. After digestion and stripping off the epidermis with a pair of forceps, the dermal tissue segment was rinsed in complete culture medium (Medium 199 or Iscove's Modified Dulbecco's Medium (IMDM)) containing 20\% foetal bovine serum (FBS), penicillin $100 \mathrm{U} / \mathrm{ml}$, streptomycin $100 \mu \mathrm{g} / \mathrm{ml}$. and 2 ul. of solubilized Amphotericin B. The scalpel blade after each scraping along the full length of the dermal segment $(n=5)$ was swirled into $5 \mathrm{ml}$. of culture medium (contained in a standing, open $25 \mathrm{ml}$. centrifuge tube) to shake off cellular matter. Finally, the centrifuge tube was capped and manually swirled and left to stand for 10 minutes. $2 \mathrm{ml}$. were pipetted out from the top and layered carefully into a pre-warmed plastic petri dish containing $5 \mathrm{ml}$. of complete culture medium and immediately transferred to an incubator at $37^{\circ} \mathrm{C}\left(5 \% \mathrm{CO}_{2} / 95 \%\right.$ $\mathrm{O}_{2}$ ). The remaining $3 \mathrm{ml}$. bottom contents of the centrifuge tube were treated likewise. Plastic was used a substrate because fibroblasts adhere rather quickly and since they secrete their own growth factor(s) (bFGF) and an extracellular matrix (ECM), no other supplements were deemed necessary.

After 8 - 12 hours, the medium was pipetted off, rinsing the cell layer with Dulbecco's phosphate buffered saline (DPBS) ( $\mathrm{pH}$ 7.4) followed and a new aliquot of complete culture medium (with no Amphotericin B) was added. After every 2 - 3 days, the culture medium was changed. To state the obvious: since the fibroblasts are secreting their own growth factor(s), a relaxed period of 2 - 3 days was privately thought to be more advanta- geous to the growing cells. Confluence was routinely obtained in 9 - 10 days without enzyme use and centrifugation.

\subsection{Sub-Culturing with a Non-Enzymatic Technique}

The fibroblast layer at confluence could be retracted using $5 \mathrm{mM}$ EDTA/DPBS ( $\mathrm{pH}$ 7.4), leaving behind a wholly intact fibroblast extracellular matrix (ECM) which can be safely stored with a layered aliquot of 5 $\mathrm{mM}$ EDTA/DPBS overnight $[12,13]$ in the incubator, if other cell experiments are envisaged. If 2 - 3 times the volume of complete culture medium were added (to neutralise the EDTA), the retracted layer (not broken into single cells but into small cell clusters) could be transferred to a T-75 flask and left overnight in the incubator for the cells to adhere at their own pace. In the morning, all medium was quickly pipetted out and about $8 \mathrm{ml}$. of DPBS were used to rinse the attached cells, before feeding with complete culture medium. Obtaining the $9-10$ day period to confluence again and normal phenotypic morphology without any cell debris proved that the fibroblasts were unaffected by the $5 \mathrm{mM}$ EDTA/DPBS treatment.

2 mM EDTA (Ethylene diamine tetra-acetic acid) was used by Rosen and Culp (1977) [14] and later by Kramer et al. (1980) [15] to induce the detachment of a monolayer of calf bovine aortic endothelial cells from the subendothelial ECM leaving behind an intact ECM. Over the years, it has become accepted as a non-enzymatic method of detaching a wide variety of primary and secondary cells from pre-treated substrates, plastic tissue culture substrates, biopolymer surfaces/scaffolds and both hydrophobic and hydrophilic substrates. It is employed when dissolved in DPBS ( $\mathrm{pH} \mathrm{7.4).} \mathrm{Integrin} \mathrm{inter-}$ action with ECM proteins is dependent on the presence of bivalent cations which EDTA chelate thereby inducing cell detachment (Li et al. 2005) [16]. The detached cells can be reseeded and will grow to confluence in an unaffected manner with normal phenotypic morphology. However, unlike with trypsin/EDTA digestion, cell counts are difficult because the cells will not detach as single cells, but as small cell clusters.

\section{RESULTS AND DISCUSSION}

Figure 1 shows dermal fibroblasts within 8 - 12 hours of cell seeding.

Figure 2 shows a sub-confluent layer of dermal fibroblasts. The pink bodies were the result of testing for microvascular endothelial cells using the diacetylated low density lipoprotein (Di-Ac-LDL) label [17]. None were found under fluorescence microscopy, using a Nikon Diaphot phase contrast microscope equipped with a reflected light fluorescence system. 


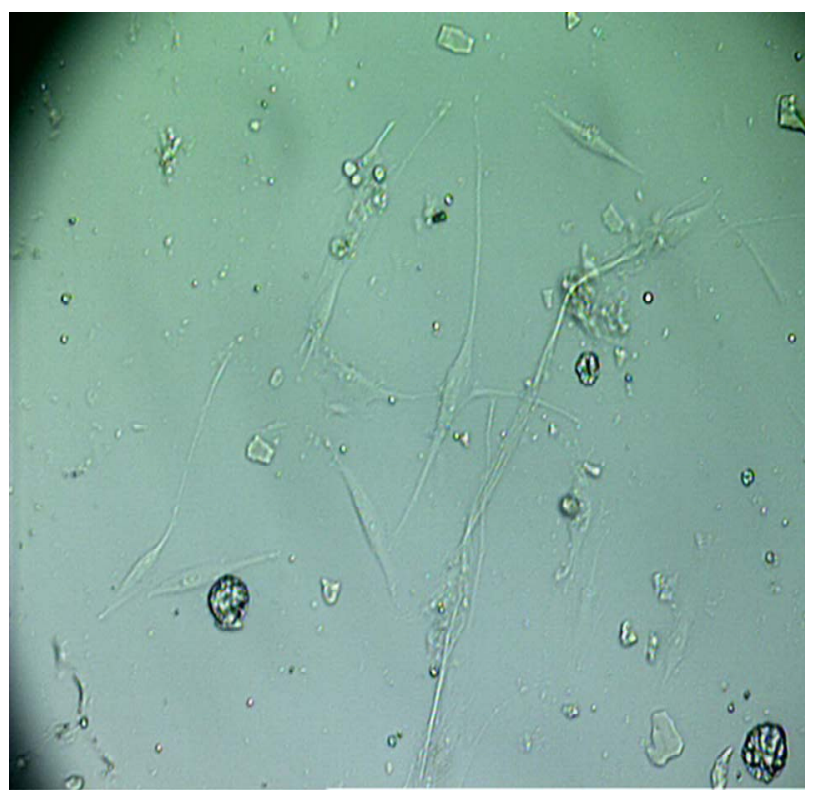

Figure 1. Within hours of initial cell plating, dermal fibroblasts can be observed. Floating basal layer keratinocytes and tissue debris can be seen; these do not adhere and are removed during the first culture medium change. Magnification: $\times 100$.

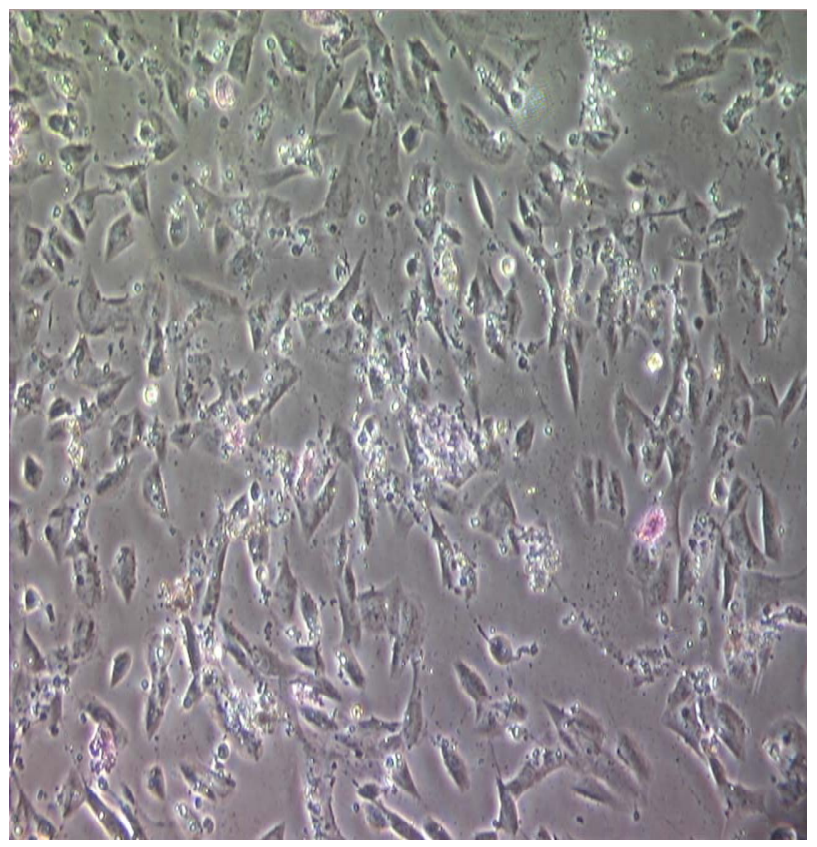

Figure 2. A subconfluent layer of reticular fibroblasts. The pink bodies seen are a result of using the Di-Ac-LDL label [17] to determine if there were any microvascular endothelial cells present. None were found using fluorescence microscopy. Magnification: $\times 100$.

Figures $\mathbf{3}$ and $\mathbf{4}$ are two different views of a confluent layer of dermal reticular fibroblasts showing their signature micro pattern with a side-on packing alignment (obtained from the lower $3 \mathrm{ml}$.). The confluent layer seemed to grow from a base tapering on both sides upwards

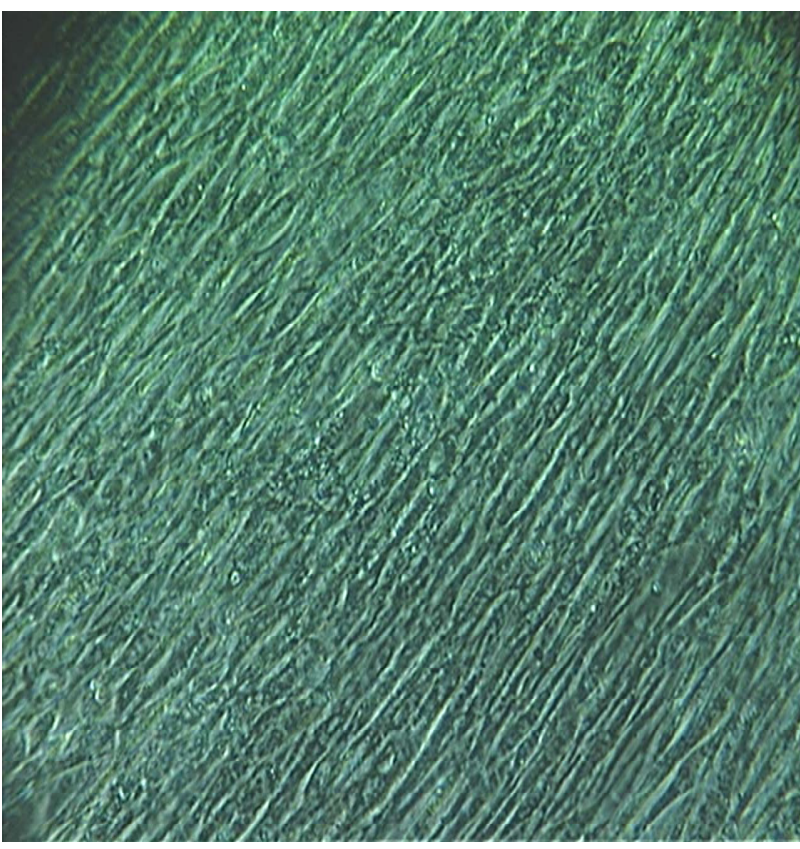

Figure 3. Dermal reticular fibroblasts at confluence. Their sideon signature packing alignment at an angled vertical is clearly visible. Magnification: $\times 100$.

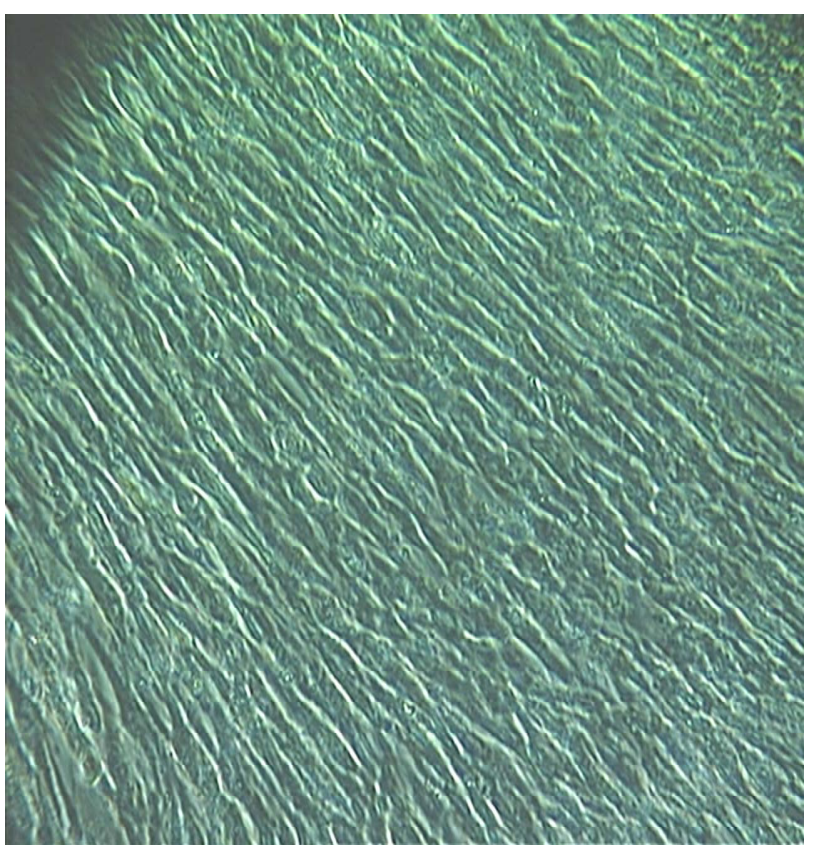

Figure 4. Another view of dermal reticular fibroblasts at confluence. Magnification: $\times 100$.

reminiscent of the broad base of a tree tapering upwards on both sides to its trunk (sadly, not photographed). Admittedly, there might have been a minute percentage of papillary fibroblasts obtained from the first scraping $(\sim 0.3 \mathrm{~mm}$ down; the reticular dermis is a further $0.7 \mathrm{~mm}$ deeper) [3], which may have settled down to the bottom of the centrifuge tube. Fibroblasts and population subsets 
of fibroblasts lack specific cell markers. It is unknown what secretory contribution or influence on, the final packing configuration was derived from papillary fibroblasts, if they were present at all.

Sutherland et al. (2005) [18] published one of the few papers which employed a non-enzymatic method of dermal tissue maceration to isolate fibroblasts. They did not realise that they were conducting a co-culture of papillary and reticular fibroblasts. Their culture medium was simply, 25 mM Hepes-buffered Hams F10 supplemented with $20 \%$ foetal calf serum, 100 units $/ \mathrm{ml}$. penicillin and $100 \mu \mathrm{g} / \mathrm{ml}$. streptomycin. They did not make particular mention of the fibroblast ECM and did not name the enzyme used for sub-culture. The ECM secreted by the cultured fibroblasts at confluence would have completely overlaid the Dow Corning flask plastic substrate they used to seed their cells on initially, and would directly determine the fibroblast configurations at confluence [19]. Hence, their monolayer at confluence (their Figure 1C [18]) shows two populations of dermal fibroblasts aligned at right angles to one another. Nevertheless, a simple deduction can be made, taking my own Figures 3 and $\mathbf{4}$ into consideration. Their vertically aligned subset of fibroblasts is being identified as reticular fibroblasts while their horizontally aligned fibroblasts as papillary fibroblasts.

Unlike their methodology, which took 6 weeks to obtain a confluent monolayer from macerated dermal tissue, the technique described herein can be easily done within 9 - 10 days. A shortened period to confluence can be attained if scrapings from several segments of autologous dermal tissue are pooled, prior to cell plating. In other words, a high density cell seeding will be executed. This is of particular importance when a cell culture of papillary fibroblasts is desired. The author found that a single scraping with the scalpel blade did not yield sufficient cellular matter to successfully proceed from the top $2 \mathrm{ml}$. aliquot. The conclusion reached was that some unknown threshold quantity of cells was required as the papillary fibroblasts take longer to attach compared to the reticular fibroblasts (substantially obtained from 4 strokes of the scalpel blade) using my bare bones cell culture technique.

\subsection{Comments on Published Wound Healing Cell Culture Studies}

The cell culture methodology described herein has implications for wound healing studies. Plucking just three publications out of the published literature [20-22] leads to the realization that conclusions drawn from wound healing studies using fibroblast preparations from minced dermis using a standardized protocol, takes hours (even overnight) of manipulation and involves upturning flasks, prior to cell plating using Gentamycin (see [5] for critical comment), may be faulty due to the use of now outdated methodology. Brem et al. 2008 [20] ascribe their experimental platform to published material by other authors $[21,22]$ which can be now updated. Scrapings from chronic wounds need to be re-examined; the authors claim they are maintaining the in vivo phenotype from primary cultures of fibroblasts grown from chronic ulcers. This is fanciful. The scrapings should have been shaken off with a gentle swirl into an aliquot of prewarmed complete culture medium containing penicillin $100 \mathrm{U} / \mathrm{ml}$., streptomycin $100 \mu \mathrm{g} / \mathrm{ml}$. and a hint of solubilized Amphotericin B ( 2 ul.) contained in a plastic petri dish and immediately incubated at $37^{\circ} \mathrm{C}$. If no cells had attached after 1 - 2 days, I would have verified there was no contamination and simply "topped up" with an aliquot of pre-warmed complete culture medium to replace evaporation loss and left the petri dish untouched in the incubator for another $1-2$ days. There is nothing testing and challenging as the authors contend. The same means can be used for scrapings from venous, pressure and diabetic foot ulcers. It is questionable whether the cellular photomicrographs of "Location A and Location C" fibroblasts (their Figure 1 [20]) do show the "phenotype of normal healthy fibroblasts" as the authors have stated. They also did not test for microvascular endothelial cells, as in Figure 2 in this paper.

What is of particular worry is that some trypsinized fibroblasts are being stored in an American national cell repository for distribution to other researchers [20]. Trypsin digestion was done, by rinsing with Puck's saline/EDTA followed by Puck's saline/Trypsin/EDTA. The authors seemed blissfully unaware that overuse of EDTA, a chelating agent, could affect cellular physiology having a direct effect on resultant cellular morphology [23]. Fine details matter a great deal in cell culture.

Researchers are advised to check the pedigree of their human dermal fibroblasts before use. Enzymatic digestions of the epidermis are being done with various enzymes and at various temperatures, e.g. $4^{\circ} \mathrm{C}$ : Sorrell et al. (2006) [24]; $12^{\circ} \mathrm{C}$ : Normand and Karasek (1995) [6]; $37^{\circ} \mathrm{C}$ : Solomon (2002) [12]. Also different enzymes are being used to digest the dermis as a shortcut to obtaining a mixed population of papillary and reticular fibroblasts. Consideration also needs to be given to flask coatings. In the author's opinion, plastic substrates of flasks and petri dishes are quite sufficient. Techniques do need to be become standardized.

Clark 1996 [25] has opined that subcutaneous tissue regeneration needs proper cell orientation and migrational cues as cellular populations change at different phases of wound healing [26]. The importance of cellular micro patterns was unknown in the 1990s. In vitro cell studies are of particular importance, if correctly conducted, to 
understand the basic behaviour and science of cells before contemplating experimental animal studies. The signature packing morphology of dermal reticular fibroblasts presents an experimental platform even while subconfluent (Figure 2). Various sample populations of agematched mesenchymal stem cells could be introduced to assess a threshold quantity and what cellular effect, if any, they would exert, having prior knowledge of the expected morphology of the confluent monolayer [see 27].

\subsection{Anatomic Site Identity Code}

A question that can be put forward for consideration is whether my reference to a 'cellular micro pattern', here and elsewhere [5], is actually a reflection of anatomic positional identity with dermal reticular fibroblasts in this instance, adopting a specific cellular packing arrangement, indicative of some sort of site identity code for dermal tissue only, which is recognized as a unique tissue pattern within the functioning human body and leads to specific fibroblast gene pattern expression in the reticular dermis and nowhere else [28]. Specific fibroblast gene pattern expressions have been described for papillary and reticular fibroblasts [29]. This reasoning could explain why the papillary fibroblasts are aligned with another cellular arrangement at right angles (a different dermal tissue site identity code) to the reticular fibroblasts in the human dermis.

The use of an autologous skin graft (split- or fullthickness) does represent the site transfer of a specific fibroblast gene pattern from the donor site to the recipient site. No consideration has been given to this fact which might provide one of the reasons why on certain occasions, the graft refuses to "take". For the use of autologous fibroblasts as a dermal filler to cosmetically repair skin defects, a published report speaks of using proprietary techniques, hence no described protocol for the expansion of fibroblast cell lines [30]. There is no long term evidence of the safety of this procedure [11]. Two questions arise. Firstly, would it be clinically advantageous to use a primary cell mixture of papillary and reticular dermal fibroblasts isolated and passaged (subcultured) non-enzymatically as is described in this paper? The second question is whether this is at all a safe procedure in the long term, given the site transfer of specific fibroblast gene pattern expressions (if both papillary and reticular fibroblasts are employed).

\subsection{Future Studies}

Normal skin cells are not exposed to serum factors generated by wounding but are bathed in a plasma filtrate [31]. It would be of interest to repeat the fibroblast cell culture described herein with both autologous plasma and serum from a patient to determine whether the same side-on confluent packing arrangement constituting the signature micro pattern would persist. Further scraping, deeper within the dermis with five-stroke increments using the scalpel blade should reveal population subsets of dermal fibroblasts [32]. Researchers must remember to test for the presence of microvascular endothelial cells.

\section{CONCLUSION}

The cellular micro pattern organization of the un-manipulated reticular fibroblasts at confluence is really striking and they appear to be absolutely natural and healthy in their microenvironment. The presence of microvascular endothelial cells was tested, but found to be absent.

From past published material [18], it was known that in cell culture, populations of papillary and reticular dermal fibroblasts are aligned at right angles to each other. Therefore, the confluent configuration of dermal fibroblasts displaying a signature micro pattern at an angled vertical was identified as reticular fibroblasts, since different populations of dermal fibroblasts lack specific cell markers. Whether this insight can be extrapolated to the in situ dermal cellular configuration is a tantalising question, which should be further explored.

A library of aging cellular profiles can now be properly constructed in an orderly, stepwise fashion from infancy to old age for both the human epidermis [5] and the dermis, if the relevant fresh skin tissue segments were obtained (compare [33]).

Footnote: Taken from a granted 2011 UK patent to the author [34].

An intact speck of epidermis remained un-noticed after stripping off the epidermis following Dispase digestion. The dermis was scraped five times with a scalpel blade. Prior to anchoring the dermal tissue in setting up a classic explant cell culture, the area around the final anchorage site in the plastic petri dish was gently swabbed with scraped surface of the tissue segment. "There was an outgrowth of two distinct morphological types of dermal fibroblasts. Dermal fibroblasts with cell-cell interlinking 'tubular connectors' organized first. This type of fibroblast is observed only in explant cultures of dermal skin tissue. The other type of dermal fibroblast, the more common morphologic type observed in primary cultures, subcultures and after enzyme digestions, consisted of a 'master' fibroblast with a thin lengthy 'proboscis' to which other fibroblasts with a much shorter proboscis connected almost at right angles (Figure 1)". (Only the mid-section of the skin tissue segment was scraped).

\section{ACKNOWLEDGEMENTS}

The University of Miami School of Medicine in Miami, Florida is 
again thanked for hosting the author as a visiting, unpaid scientist during the summer of 2001 .

\section{REFERENCES}

[1] MacNeil, S. (2007) Progress and opportunities for tissueengineered skin. Nature, 445, 874-880. http://dx.doi.org/10.1038/nature05664

[2] McDougall, S., Dallon, J., Sherratt, J. and Maini, P. (2006) Fibroblast migration and collagen deposition during dermal wound healing: Mathematical modelling and clinical implications. Philosophical Transactions of the Royal Society A, 364, 1385-1405.

http://dx.doi.org/10.1098/rsta.2006.1773

[3] Sorrell, J.M. and Caplan, A.I. (2004) Fibroblast heterogeneity: More than skin deep. Journal of Cell Science, 117, 667-675. http://dx.doi.org/10.1242/jcs.01005

[4] Sorrell, J.M., Baber, M.A. and Caplan, A.I. (2004) Sitematched papillary and reticular human dermal fibroblasts differ in their release of specific growth factors/cytokines and in their interaction with keratinocytes. Journal of Cellular Physiology, 200, 134-145. http://dx.doi.org/10.1002/jep.10474

[5] Solomon, D.E. (2012) Mimicry of a natural, living intraepidermal micro pattern used in guided tissue regeneration of the human epidermis. Journal of Developmental Biology and Tissue Engineering, 4, 1-7.

[6] Normand, J. and Karasek, M.A. (1995) A method for the isolation and serial propagation of keratinocytes, endothelial cells, and fibroblasts from a single punch biopsy of human skin. In Vitro Cellular \& Developmental Biology: Animals, 31, 447-455.

http://dx.doi.org/10.1007/BF02634257

[7] Pandamooz, S., Hadipour, A., Akhavan-Niaki, H., Pourghasem, M., Abedian, Z., Ardekani, A.M., Golpour, M., Hassan, Z.M. and Mostafazadeh A. (2012) Short exposure to collagenase and coculture with mouse embryonic pancreas improve human dermal fibroblast culture. Biotechnology and Applied Biochemistry, 59, 254-261. http://dx.doi.org/10.1002/bab.1020

[8] Pageon, H., Zucchi, H. and Asselineau, D. (2012) Distinct and complementary roles of papillary and reticular fibroblasts in skin morphogenesis and homeostasis. European Journal of Dermatology, 22, 324-332.

[9] Sandulache, V.C., Zhou, Z., Sherman, A., Dohar, J.E. and Hebda, P.A. (2003) Impact of transplanted fibroblast on rabbit skin wounds. JAMA Otolaryngology: Head \& Neck Surgery, 129, 345-350.

http://dx.doi.org/10.1001/archotol.129.3.345

[10] Schönherr, E., Beavan, L.A., Hausser, H., Kresse, H. and Culp, L.A. (1993) Differences in decorin expression by papillary and reticular fibroblasts in vivo and in vitro. Biochemical Journal, 290, 893-899.

[11] Munavalli, G.S., Smith, S., Maslowski, J.M. and Weiss, R.A. (2013) Successful treatment of depressed, distensible acne scars using autologous fibroblasts: A multi-site, prospective, double blind, placebo-controlled clinical trial. Dermatologic Surgery, 39, 1226-1236. http://dx.doi.org/10.1111/dsu.12204
[12] Solomon, D.E. (2002) An in vitro examination of an extracellular matrix scaffold for use in wound healing. International Journal of Experimental Pathology, 83, 209216. http://dx.doi.org/10.1046/j.1365-2613.2002.00238.x

[13] Korner, G., Bjornsson, T.D. and Vlodavsky, I. (1993) Extracellular matrix produced by cultured corneal and aortic endothelial cell contains active tissue type and urokinasetype plasminogen activators. Journal of Cellular Physiology, 154, 456-465. http://dx.doi.org/10.1002/jep.1041540303

[14] Rosen, J.J. and Culp, L.A. (1977) Morphology and cellular origins of substrate-attached material from mouse fibroblasts. Experimental Cell Research, 107, 139-150. http://dx.doi.org/10.1016/0014-4827(77)90395-0

[15] Kramer, R.H., Gonzalez, R. and Nicholson, G.L. (1980) Metastatic tumor cells adhere preferentially to the extracellular matrix underlying vascular endothelial cells. International Journal of Cancer, 26, 639-645. http://dx.doi.org/10.1002/ijc.2910260516

[16] Li, J., Thielemann, C., Reuning, U. and Johannsmann, D. (2005) Monitoring of integrin-mediated adhesion of human ovarian cancer cells to model protein surfaces by quartz crystal resonators: Evaluation in the impedance analysis mode. Biosensors and Bioelectronics, 20, 13331340. http://dx.doi.org/10.1016/j.bios.2004.05.004

[17] Voyta, J.C., Via, D.P., Butterfield, C.E. and Zetter, B.R. (1984) Identification and isolation of endothelial cells based on their increased uptake of acetylated low density lipoprotein. Journal of Cell Biology, 99, 2034-2040. http://dx.doi.org/10.1083/jcb.99.6.2034

[18] Sutherland, J., Denyer, M. and Britland, S. (2005) Contact guidance in human dermal fibroblasts is modulated by population pressure. Journal of Anatomy, 206, 581587. http://dx.doi.org/10.1111/j.1469-7580.2005.00415.x

[19] Sorrell, J.M. and Caplan, A.I. (2009) Fibroblasts-A diverse population at the center of it all. International Review of Cell and Molecular Biology, 276, 161-214. http://dx.doi.org/10.1016/S1937-6448(09)76004-6

[20] Brem, H., Golinko, M.S., Stojadinovic, O., Kodra, A., Diegelmann, R.F., Vukelic, S., Entero, H., Coppock, D.L. and Tomic-Canic, M. (2008) Primary cultured fibroblasts derived from patients with chronic wounds: A methodology to produce human cell lines and test putative growth factor therapy such as GMCSF. Journal of Translational Medicine, 6, 75 . http://dx.doi.org/10.1186/1479-5876-6-75

[21] Brem, H., Stojadinovic, O., Diegelmann, R.F., Entero, H., Lee, B., Pastar, I., Golinko, M., Rosenberg, H. and Tomic-Canic, M. (2007) Molecular markers in patients with chronic wounds to guide surgical debridement. Molecular Medicine, 1, 30-39. http://dx.doi.org/10.2119/2006-00054.Vittorini

[22] Egles, C., Shamis, Y., Mauney, J.R., Volloch, V., Kaplan, D.L. and Garlick, J.A. (2008) Denatured collagen modulates the phenotype of normal and wounded human skin equivalents. Journal of Investigative Dermatology, 128, 1830-1837. http://dx.doi.org/10.1038/sj.jid.5701240

[23] Vogel, K.G. (1978) Effects of hyaluronidase, trypsin, and EDTA on surface composition and topography during detachment of cells in culture. Experimental Cell Research, 
113, 345-357.

http://dx.doi.org/10.1016/0014-4827(78)90375-0

[24] Sorrell, J.M., Baber, M.A., Caplan, A.I. (2006) Clonal characterization of fibroblasts in the superficial layer of the adult human dermis. Cell and Tissue Research, 327, 499-510. http://dx.doi.org/10.1007/s00441-006-0317-y

[25] Clark, R.A.F. (1996) Wound repair: Overview and general considerations. In: Clark, R.A.F., Ed., The Molecular, Cellular Biology of Wound Repair, Plenum Press, New York, 3-35.

[26] Singer, A.J. and Clark, R.A.F. (1999) Cutaneous wound healing. The New England Journal of Medicine, 341, 738-746. http://dx.doi.org/10.1056/NEJM199909023411006

[27] Sorrell, J.M. and Caplan, A.I. (2010) Topical delivery of mesenchymal stem cells and their function in wounds. Stem Cell Research \& Therapy, $\mathbf{1}, 30$. http://dx.doi.org/10.1186/scrt30

[28] Rinn, J.L., Bondre, C., Gladstone, H.B., Brown, O., Chang, H.Y. (2006) Anatomic demarcation by positional variation in fibroblast gene expression. PLoS Genetics, 2, e119. http://dx.doi.org/10.1371/journal.pgen.0020119

[29] Janson, D.G., Saintigny, G., Van Adrichem, A., Mahé, C. and El-Ghalbzouri, A. (2012) Different gene expression patterns in human papillary and reticular fibroblasts. Journal of Investigative Dermatology, 132, 2565-2572. http://dx.doi.org/10.1038/jid.2012.192

[30] Watson, D., Keller, G.S., Lacombe, V., Fodor, P.B., Rawnsley, J., Lask, G.P. (1999) Autologous fibroblasts for treatment of facial rhytids and dermal depressions. A pilot study. Archives of Facial Plastic Surgery, 1, 165-170. http://dx.doi.org/10.1001/archfaci.1.3.165

[31] Li, W., Fan, J., Chen, M., Guan, S., Sawcer, D., Bokoch, G.M. and Woodley, D.T. (2004) Mechanism of human dermal fibroblast migration driven by Type 1 collagen and platelet-derived growth factor-BB. Molecular Biology of the Cell, 15, 294-309. http://dx.doi.org/10.1091/mbc.E03-05-0352

[32] Novotny, G.E.K. and Gnoth, C. (1991) Variability of fibroblast morphology in vivo: A silver impregnation study on human digital dermis and subcutis. Journal of Anatomy, 177, 195-207.

[33] Mine, S., Fortunel, N.O., Pageon, H. and Asselineau, D. (2008) Aging alters functionally human dermal papillary fibroblasts but not reticular fibroblasts: A new view of skin morphogenesis and aging. PLoS One, 3, e4066. http://dx.doi.org/10.1371/journal.pone.0004066

[34] Solomon, D.E. (2011) An in vitro human skin assay protocol which directly uses the natural cellular interactions between un-manipulated autologous human epidermal and dermal cells. United Kingdom Patent Gazette (Intellectual Property Office), GB2442271B. 